Mitteilungen der Schweizerischen Gesellschaft für Phlebologie

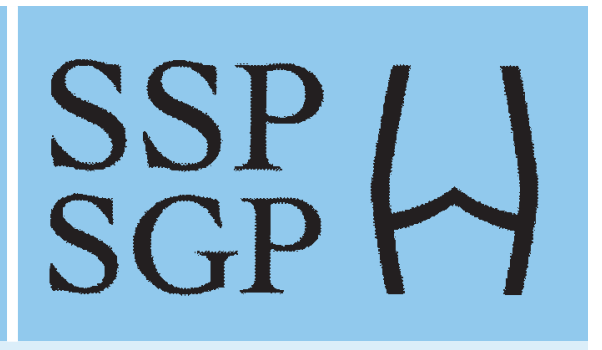

\title{
EVF virtual congress 2021 (Budapest)
}

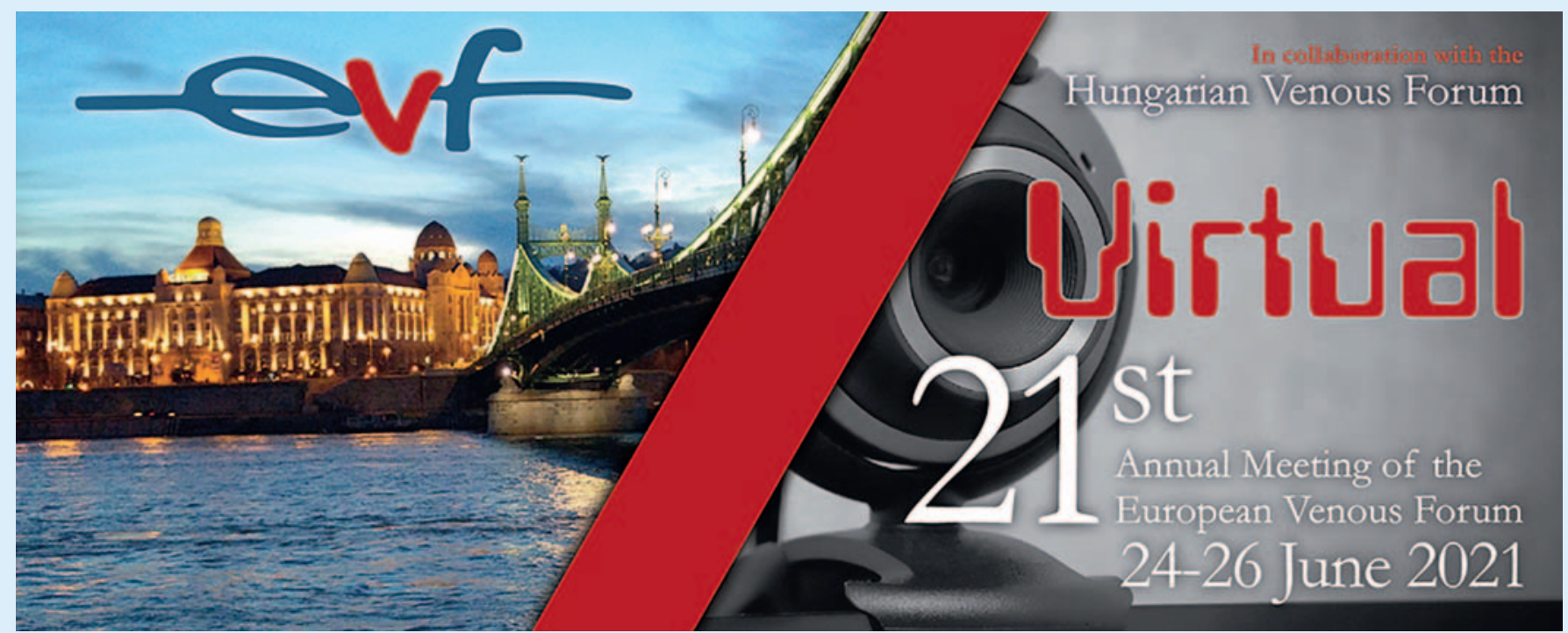

Quelle: European Venous Forum.

Dominik Heim

Es solle ein Bericht über den diesjährigen Kongress des European Venous Forum, EVF, sein, wurde mir gesagt. Also, aber der war ja so richtig anders als in früheren Jahren! 2019 konnten wir uns noch - in der Prä-Corona-Ära - in der Universität in Zürich treffen - wie immer. Der Hörsaal war voll, man besuchte die Industriestände, man diskutierte, machte Small Talk und genoss die Gelegenheit sich (wie vielleicht schon im Vorjahr) wiederzutreffen. Zürich war heiß, sehr heiß (nicht nur die Themen), es war der heißeste Kongress des EVF, dabei war er doch auch schon früher in Athen und anderen südlichen Städten.

Dann kam Corona, eben noch war das EVF Gast auf dem Jahreskongress des American Venous Forum, AVF, auf Amelia Islands (4.6. März 2020). Die Präsidenten, Chairmen und Entourage trafen sich und beschlossen, in Zukunft etwas enger zusammenzuarbei- ten. Denn auch wenn ein Atlantik dazwischen liegt, sind sich die venösen Probleme auf beiden Seiten doch sehr ähnlich. Ein MOU (Memorandum of Understanding) wurde erarbeitet und von den beiden Boards dann gutgeheißen, man stellte sich auf die Transatlantikflüge ein und freute sich auf das Wiedersehen.

Corona stoppte diesen Prozess. Der EVFJahreskongress in Budapest 2020 wurde auf 2021 verschoben, und die digitale Kongresswelt nahm ihren Anfang. Das EVF tat sich mit Shocklogic, einer Software-Firma in London, zusammen, und unter der tatkräftigen Leitung von Anne Taft, Executive Director des EVF, Dawn Bond, Exhibition Manager, und Karen Marwood-Jones wurde der ganze Kongress virtuell geplant. Das wissenschaftliche Programm wurde in unzähligen Mittwochmittag-Zoom-Konferenzen von Andrew Nicolaides aus Zypern, Chairman EVF, Imre Bihari aus Budapest, Incoming President und Congress President,
Niels Baekgaard aus Kopenhagen, EVF Board Member, und mir erarbeitet. Trotz meiner anfänglichen Skepsis funktionierte dieses Format der (Zoom-) Kongressplanung erstaunlich gut. Man musste sich halt die Zeit nehmen. Shocklogic erwies sich - trotz meiner anfänglichen Skepsis - zu einem zuverlässigen Partner; das sollte sich dann bei der „Ausstrahlung“ des Kongresses nochmals zeigen.

Am EVF-typischen Format des Kongresses wurde festgehalten: Abstract Sessions - 10 Minuten Präsentation und 10 Minuten Diskussion, etwas weniger animiert und emotional, als wenn live -, die besten 28 wurden dafür aus über 100 Einsendungen aus ganz Europa ausgewählt: Didactic Sessions, Industry Sessions, E-Poster Sessions (28 Papers), Prize Winning Session und die in Zürich 2019 erstmals durchgeführte EVF-AVF Joint Session. Die Invited Lecture wurde von Peter Gloviczki, Rochester, USA, unter dem Titel „The best vein care is the only 
vein care to be considered" gehalten. Das venöse Spektrum war - wie immer - breit, eine eigentliche Revolution in den Behandlungsoptionen und -strategien des oberflächlichen und tiefen Systems blieb aber aus, man modifizierte, optimierte und erprobte die schon bekannten Techniken. Zum Glue kam ein russisches Fabrikat dazu, der Stellenwert der Vasoaktiva kam zur Sprache (notabene auch in der Behandlung von COVID-Patienten) und eine klare, allgemeinumfassende Strategie in der Behandlung von DVT und vom postthrombotischen Syndrom wurde gesucht. In der sportlichen Welt würde man von einem hochkarätigen Meeting ohne Weltrekord sprechen.

Und wie schon angetönt, lag die Herausforderung diesmal nicht nur in der wissenschaftlichen Ebene, sondern auch in der digitalen Form. Die Jungen waren da deutlich versierter, und die „Alten“ hatten nicht selten einen jungen Mitarbeiter „hinter dem Vorhang" (ich auch). Es hat sich bewährt, dass man vorgängig an den Kongress eine Videoversion seines Vortrags einsenden musste, auf die bei technischen Schwierigkeiten zurückgegriffen werden konnte. Die dann wirklich live präsentierten Vorträge waren im Vergleich zu den durchweg videoisierten Vorträgen des AVF (nur ein Beispiel) viel lebendiger und authentischer. Fragen der „Delegates“ konnten aber nicht live, sondern nur über die Moderatoren schriftlich gestellt werden - so blieben verbale „Auseinandersetzungen“, die diese Kongresse beleben können, in diesem Format aus. Auch den Kaffee in den Pausen holte man in der eigenen Küche und nicht am Stand der Aussteller. Diese hatten aber trotzdem ihre eigenen Booths, in die man „hineingehen“ konnte, das Praliné oder die Luxemburgerli musste man sich virtuell auf der Zunge zergehen lassen. Dass die Aussteller trotz dieses neuen Formats ausstellten, wird jetzt in der finanziellen Bilanz sichtbar - man schloss (wie immer bei den EVF-Kongressen der letzten Jahre) auch dieses Jahr mit einem Gewinn.

Für all jene, die nicht die Zeit und Muße hatten, 3 Tage vor dem Bildschirm zu sitzen, sei hier am Rande erwähnt, dass die Vorträ- ge bei erfolgter Registration während eines Jahres nach dem Kongress noch nachgeschaut werden können.

Unterdessen laufen die Vorbereitungen für den nächsten Jahreskongress in Venedig (30. Juni bis 2. Juli 2022), man diskutiert eine Hybridversion, da wohl nicht jeder auf's Vaporetto auf dem Canal Grande will oder den überteuerten Caffé auf der Piazza San Marco trinken will; übrigens kann man Venedig auch virtuell in einem Buch von Donna Leon genießen (das neueste „Transient Desires“ ist soeben erschienen). Mit welchem Roman man dann die virtuelle Ambiance von Berlin (22.-24. Juni 2023) einfangen will, ist noch nicht entschieden.

Goodbye Budapest, wir hätten Dich gerne live erlebt!

PS: Mehr Details (Programm, Prize Winners etc.) sind auf www.europeanvenousforum. org nachzulesen. 